\title{
Analytical and numerical analysis of a "springback-forming" process dedicated to stiffened panels
}

\author{
M. E. Ait ali ${ }^{\mathrm{a}, \mathrm{b}, *}$, D. Guines ${ }^{\mathrm{a}}$, L. Leotoing $^{\mathrm{a}}$, E. Ragneau $^{\mathrm{a}}$ \\ ${ }^{a}$ Université Européenne de Bretagne, INSA de Rennes - LGCGM, \\ 20, avenue des buttes de coesmes, 35708 Rennes cedex 7, France \\ ${ }^{b}$ Université Mohammed V, Ecole Mohammadia d'Ingénieurs - ERD3M, \\ 20, avenue Ibnsina, 10010 Rabat, Morocco
}

\begin{abstract}
The aim of this article is to present and to analyze the capabilities of a process named "springback-forming", dedicated to stiffened panels such as airplane's fuselage panels. The principle of this forming process is to apply a tension on the stiffener, before the assembly stage with the sheet in a flat configuration using fasteners, adhesives, or a welding process... the bending of the structure is then achieved by springback energy of the stiffener when its tension is released. Using an analytical and finite element models, we studied the capabilities of this process in terms of curvature limits in the case of a single-curved stiffened panel. The results of both models are in good agreement. Through a parametric study, numerical simulations show that when the structure is relatively slender the curvature radius obtained is uniform. Moreover, the value of this radius is independent of the structure's length and is mainly limited by the stiffener's height. The carried out experimental tests, using laser beam welding as a joining process, demonstrated the feasibility of the process. From the proposed modeling, it is possible to evaluate the range of achievable curvature radius and its uniformity for different values of both geometrical and mechanical parameters of the structure.
\end{abstract}

Keywords: Stiffened panel, Forming process, Single-curved panel, Springback-forming

\footnotetext{
*Principal corresponding author

Email address: aitali@emi.ac.ma, Mobile +212654126898 (M. E. Ait ali)
} 


\section{Introduction}

The transportation sector, including aeronautics, automobiles, railway and naval, is based in a large proportion on forming metallic materials. In these sectors, there is a constant need of reducing costs such as: - product development cost (in prototyping or in industrialization stage); - tools cost by making them, for example, more reusable; - manufacturing costs by having less parts and reducing the assembly time. This constant need led to a global approach aiming to have: the most suitable manufacturing processes for each type of parts, and a robust simulation tools to analyze the performance of these processes. In this context, the airplanes manufacturers are interested in the development of innovative forming processes dedicated to stiffened panels such as fuselage panels. These structures are constructed primarily from thin sheets, called also web or skin, and stiffening elements such as beams (Megson, 2010).

An assessment of existing manufacturing technology for metallic fuselage structure was carried out by Pettit et al. (2000). We distinguish, in this assessment, two categories of manufacturing strategy of these stiffened panels: - in the first category, sheets and stiffeners are formed separately and then assembled, mostly by riveting; - in the second category, sheets and stiffeners are first assembled and then formed together to the correct shape.

In the first category, the manufacturing of each element of the structure is based on conventional processes. The most used process for sheets is roll forming to make singly curved panel, as reported by Megson (2010). This process is usually replaced by stretch forming for doubly curved or more complex panels. The stiffeners are extruded or machined and then assembled with the sheet (using bolts, rivets, or a welding process). In this category, the precision of the final shape is mainly dependent on the precision of each component. Furthermore, automatizing of such assembly operations is costly in terms of machines and tools, mainly because of the curvature of the stiffened panels. In contrast, the assembly in flat configuration requires less sophisticated machines and therefore is more cost effective and easier to control.

In the second category, press bend-forming is an effective and often used process, as reported in NASA-CR-124075 (1973). On the one hand, the main advantage of this process is the use of a universal die for all panels; on the 
other hand, the key problem is the design of the forming path of the punch used in the bending process. This issue is often solved by using finite element models instead of an experimental approach. Because of the time consuming simulations, Yan et al. (2009) developed an equivalent model to improve the efficiency of the finite element model and optimize the bend forming path. Moreover, the cost of this process increases because of the considerable realignment work needed to achieve the imposed tolerances (Meyer et al., 1987). A more versatile process, in the same category, with lower machine and manufacturing costs, is shot peen-forming. This process is a major process for manufacturing wing skins (Wang and Platts, 2002) and is also used successfully to form fuselage panels (Meyer et al., 1987). Its versatility comes from its adaptability to all panel sizes, reduced machines costs since neither the die nor the punch is needed, and its good production rate. However, with this process only small curvature is achievable and special precautions are necessary to avoid producing doubly curved panel. Li (1981) studied experimentally the use of pre-bending of the panel while it is formed, using peen-forming, as a way to form single-curved stiffened panel. He showed that the increase of the pre-bending loads induce the decrease of the curvature radius in the pre-bending direction and the increase of the curvature radius in the perpendicular direction. Similarly to other processes, to determine the process parameters, the trial-and-error approach is more and more replaced with efficient numerical models. Wang and Platts (2002) presented a numerical procedure to obtain the initial blank shape from the final formed surface. Gariepy (2012) developed a finite element model of the process capable of predicting accurately the final shape and the effect of different parameters on the process.

A variation of press bend-forming is warm forming. In this process, the bending capability of the panel is extended by increasing the working temperature, during forming, for an adequate amount of time. Generally, the working temperature is around 200 to $300^{\circ} \mathrm{C}$ for aluminum alloys (Toros et al., 2008). The warm temperature increases the material ductility and lowers its yield strength. As a result, smaller curvature radii are achieved compared with cold forming processes. However, because of the warming equipment necessary additional cost is added.

A more favored process, in the aerospace industry, is creep age-forming (Lin et al., 2006). In this process a heat treatment (artificial aging of aluminum alloys like the 2000 series) takes place, in an autoclave, simultaneously with the forming process. The latter is a bend-forming process using vacuum bagging 
technique. Holman (1989) showed, by experience, that the smallest residual stresses are obtained using creep age-forming, compared with roll forming, press bend forming, and shot peen-forming. Brewer (1989) tested successfully its feasibility in the case of wing skins and fuselage panels. However, with this process a large springback occurs. An exploratory experimental work was led by Airbus Saint-Nazaire to form a single-curved stiffened panel using this process. The springback varies from 65 to $90 \%$. To help predict the curvature achievable and control the springback, robust numerical models are more and more used. In addition, these models serve to study the feasibility of applying creep age-forming to stiffened panels. Lin et al. (2006) developed a numerical model to estimate the springback of a non-stiffened sheet. The results are between 65 and $80 \%$ of the tool's radius. Takafumi et al. (2004) studied experimentally and numerically the forming of doubly-curved stiffened wing skins (using creep age forming). The springback obtained is between 50 and $70 \%$ (of the tool's radius) and the difference between the two approaches is less than $7 \%$. Davoodi (2006) studied numerically the forming of a single-curved stiffened panel. The springback obtained is between 65 and $90 \%$.

In this article, we investigate the feasibility of a process that we named "springback-forming". The proposed process: - belongs to the second category; - does not need a die or a punch as in shot peen-forming; - the springback is absent, contrary to the processes mentioned above. To study and analyze the performance of this process, we illustrate its principle in the case of the forming of a single-curved panel with one stiffener. We also developed an analytical and a numerical model to determine the capabilities and the limitations of the process in terms of the achievable curvature radius. We find a good agreement between the two models. We conducted experimental tests, using laser beam welding as a joining process, which demonstrated the feasibility of the process and found a qualitative agreement with numerical model. Hence, using the analytical tool we can evaluate quickly the effect of various parameters on the process.

\section{Principle and analytical analysis of springback-forming}

The objective of this section is to describe the principle of the process and to analyze it using an analytical model. This analytical model allows us to study the effect of geometrical and mechanical parameters on the final shape, and on the residual stresses in the structure after forming. 


\subsection{Principle of the process}

In order to illustrate the principle of springback-forming, we consider, in this article, the forming stages of a single-curved stiffened panel. Initially, this stiffened panel contains a rectangular plate and one straight stiffener. Both have the same length and a uniform rectangular cross-section. The stiffener is positioned symmetrically with respect to the plate's width. The springback-forming in this case is composed of three steps:

- Step 1: the stiffener is pre-loaded by a tension force $\vec{F}$ applied along its neutral axis, Fig. 1.a.

- Step 2: the stiffener and the plate are joined while the stiffener's tension is maintained, Fig. 1.b.

- Step 3: the stiffener's tension $\vec{F}$ is released, Fig. 1.c.

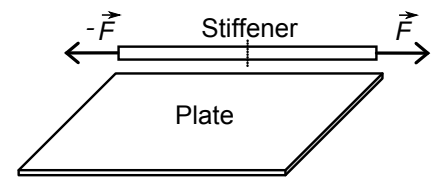

(a) Step 1: applying a tension on the stiffener.
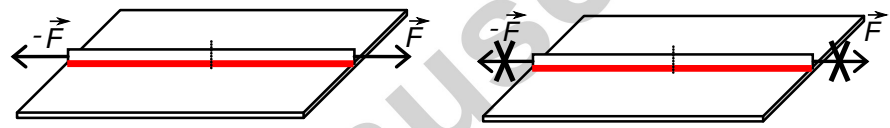

(b) Step 2: maintaining the (c) Step 3: releasing the tenstiffener's tension while as- sion. sembling it with the plate.

Fig. 1: The three steps of springback-forming.

As described above, this process does not need a die or a punch, the only tool needed is a tool to apply the tension on the stiffener, additionally all the assembly operations are done in a flat configuration. The assembly process (stiffener/plate) could be any process like riveting or welding. Neither the assembly process nor its influence on the forming process is studied in this article.

In step 3, the springback of the stiffener creates a compression load. The resultant of this load is applied in the direction of the stiffener's neutral axis. After the assembly, the centroid of the stiffened panel's cross-section changes (moves toward the plate), thus a bending moment appears in this step. This permanent bending moment allows the forming of the panel. In other words, the springback energy stored in the stiffener allows the forming of the structure. 


\subsection{Analytical model of the process}

To evaluate the curvature radius obtained by springback-forming, we develop a model based on the Euler-Bernoulli beam theory. Let's consider the case of the stiffened panel described above in Section 2.1. Fig. 2 shows different geometrical parameters of a cross-section of the assembly (stiffener and plate). Let's denote:

- $y_{T}, y_{R}$, and $y_{G}$, the y-coordinate of the cross-section centroid of the plate, the stiffener and the assembly (stiffener and plate);

- $e_{T}$ and $B$, the thickness and the width of the plate;

- $e_{R}$ and $h$, the thickness and the height of the stiffener;

- $S_{R}$ and $S_{T}$, the cross-sectional area of the stiffener and the plate.

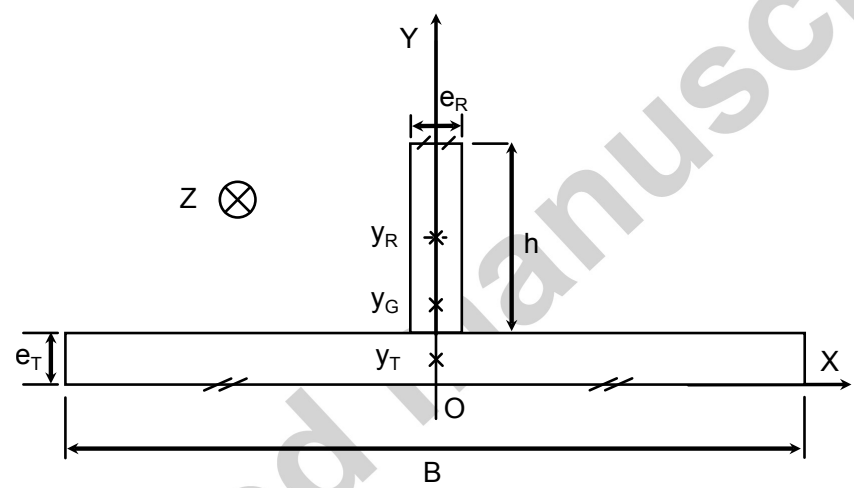

Fig. 2: Geometrical parameters of the assembly's cross-section (stiffener and plate).

In step 3, the bending moment generated is given by the expression:

$$
M_{f x}=F\left(y_{R}-y_{G}\right)
$$

In order to use the classical beam theory formulas, the following assumptions are made:

- The assembly between the plate and the stiffener is rigid so no relative displacement is allowed. 
- The assembly process does not introduce any stress field in the structure.

- The structure's cross-section dimensions are smaller than its length so that it might be considered as a beam.

- The stiffener and the plate's material are the same and this material is considered homogenous, isotropic, linear, and elastic.

- The geometrical non-linearities are neglected.

Thus, the longitudinal curvature radius (along $\mathrm{Z}$ axis) is given by

$$
R=\frac{E\left(I_{R}^{G}+I_{T}^{G}\right)}{M_{f x}}
$$

Where $I_{R}^{G}$ and $I_{T}^{G}$ are the inertia moments of the cross-section of the stiffener and the plate with respect to the axis $(G, \vec{X})$ and $E$ is Young's modulus of the stiffener and the plate.

According to this expression, the panel has a uniform longitudinal curvature.

\subsection{Geometrical analysis}

The curvature radius's expression, given by Eq. 2, makes it possible to analyze the effect of the geometrical and mechanical parameters on the process. First, we have to express it using independent parameters: $h, e_{R}$, $B, e_{T}, E$, and $\sigma=F / S_{R}$ (the tensile stress applied initially to a stiffener's cross-section). We obtain

$$
R=\frac{E\left(h^{4} e_{R}^{2}+4 h^{3} e_{R} B e_{T}+6 B e_{T}^{2} e_{R} h^{2}+4 h e_{R} B e_{T}^{3}+B^{2} e_{T}^{4}\right)}{6 \sigma h e_{R} B e_{T}\left(e_{T}+h\right)}
$$

In order to study the sensibility of the process to different geometrical parameters, we differentiate the expression given by Eq. 3 with respect to these parameters to look for optimums. Thus, we obtain

$$
\begin{aligned}
& \frac{\partial R}{\partial B}=0 \quad \Leftrightarrow \quad B\left(R_{\text {min }}\right)=\frac{h^{2} e_{R}}{e_{T}^{2}} \\
& \frac{\partial R}{\partial h}=0 \quad \Leftrightarrow \quad h\left(R_{\text {min }}\right)=\frac{e_{T}\left(\alpha^{2}+e_{R}^{2}-e_{R} \alpha\right)}{2 e_{R} \alpha} \\
& \frac{\partial R}{\partial e_{R}}=0 \quad \Leftrightarrow \quad e_{R}\left(R_{\text {min }}\right)=\frac{B e_{T}^{2}}{h^{2}} \\
& \frac{\partial R}{\partial e_{T}}=0 \quad \Leftrightarrow \quad e_{T}\left(R_{\text {min }}\right)=\frac{h\left(\beta^{2}+B^{2}-B \beta\right)}{2 B \beta}
\end{aligned}
$$


172

where

$$
\begin{aligned}
\alpha & =\left(e_{R}^{2}\left(2 B+2 \sqrt{B\left(B-e_{R}\right)}-e_{R}\right)\right)^{\frac{1}{3}} \\
\text { and } \beta & =\left(B^{2}\left(2 e_{R}+2 \sqrt{e_{R}\left(e_{R}-B\right)}-B\right)\right)^{\frac{1}{3}}
\end{aligned}
$$

These minima $\left(B\left(R_{\min }\right), h\left(R_{\min }\right), e_{R}\left(R_{\min }\right)\right.$, and $\left.e_{T}\left(R_{\min }\right)\right)$ does not always correspond to realistic stiffened panels dimensions. However, they are the reference for the minimum curvature radius achievable for any configuration. For example, if we fix all the parameters except the stiffener's height, then the smallest curvature radius achievable with this process, for this structure, is obtained when $h=h\left(R_{\min }\right)$. On the other hand, it is interesting to observe that the expression of these minima depends only on the geometry of the structure, and does not depend on the initial tensile stress, which could be useful during the design of such structures.

To further understand the existence of these minima, let's consider Eq. 2. When a parameter varies, the inertia moment of the cross-section and the bending moment (Eq. 1) are also modified. Both of these quantities depend on $y_{G}$. The expression of $y_{G}$ is given by:

$$
y_{G}=\frac{B e_{T}^{2}+2 h e_{R} e_{T}+h^{2} e_{R}}{2\left(h e_{R}+B e_{T}\right)}
$$

We note that when $B=B\left(R_{\min }\right)$ or $e_{R}=e_{R}\left(R_{\text {min }}\right)$, we have $y_{G}=e_{T}$, which means that the cross-section centroid is at the interface stiffener-plate. It is worth noting that this observation is not valid when $h=h\left(R_{\text {min }}\right)$ or when $e_{T}=e_{T}\left(R_{\min }\right)$.

Another way to demonstrate the existence of these minima is by considering the extreme values of each parameter:

1. For the plate's width $B, B \mapsto 0$ is equivalent to the case where there is no plate. After the release of the stiffener, the latter will not bend, hence $R=\infty$. Similarly, if $B \mapsto+\infty$, the plate's inertia moment approaches infinity but the bending moment $M_{f x}$ remains finite. Therefore, considering Eq. 2, $R=\infty$, which demonstrates the existence of a value of $B$ that gives a minimum value of $R$.

2. In the case of the stiffener's thickness $e_{R}, e_{R} \mapsto 0$ is equivalent to a panel without a stiffener, so the plate stays flat, thus $R=\infty$. Using the same argument when $e_{R} \mapsto+\infty$ as when $B \mapsto+\infty$, we deduce the existence of a value of $e_{R}$ that gives a minimum value of $R$. 
3. Let's consider now the extreme values of the stiffener's height $h$. $h \mapsto 0$ is equivalent to a structure with only a plate, so it stays flat, this means $R=\infty$. However, if $h \mapsto+\infty$, the bending moments $M_{f x} \mapsto 0$ according to Eq. 1. Moreover, the stiffener's inertia moment approaches infinity ( $h$ is cubed in this inertia moment), and considering Eq. 2 we deduce that $R=\infty$. In the same way, we conclude that there is a value of $h$ that gives a minimum value of $R$.

4. For the plate's thickness $e_{T}, e_{T} \mapsto 0$ is equivalent to saying that there is no plate. As in the case of the plate's width, the stiffener will not bend, so $R=\infty$. However, when $e_{T} \mapsto+\infty$, the bending moment generated will increase linearly with $e_{T}$, but the plate's inertia moment will increase more rapidly, as its expression contains the term $e_{T}^{3}$. Taking into account Eq. 2, we deduce that $R=\infty$ and hence the existence of minimal value of $R$ for a certain value of $e_{T}$.

\subsection{Mechanical analysis}

Mechanical analysis of the process concerns: (i) the effect of the material's Young's modulus on the process (ii) and investigations of stress distribution in the structure at different stages of the process.

For given fixed dimensions of the structure, according to the curvature radius expression, Eq. 2, the smaller the material's stiffness (stiffener and/or plate), the smaller the curvature radius achieved.

As for the stresses induced in the structure, when the pre-load $F$ is applied during step 1 of the process, a positive tensile stress $\sigma^{1}=F / S_{R}$ is generated in the stiffener. The plate is supposed to be initially free of stress. In step 2 , we supposed (in Section 2.2) that the assembly process does not introduce any stress field in the structure. In step 3, releasing the tension force is equivalent to applying, in both the stiffener and the plate, a compression stress $\sigma_{\text {Compr }}^{3}=-S_{R} \sigma^{1} /\left(S_{R}+S_{T}\right)$ and a bending stress $\sigma_{\text {Bend }}^{3}=-M_{f x}(y-$ $\left.y_{G}\right) /\left(I_{R}^{G}+I_{T}^{G}\right) . \quad M_{f x}$ is the bending moment given by Eq. 1 and $y$ is the y-coordinate of a point of the considered cross-section (see Fig. 2). By applying the superposition principle, we deduce the residual stress $\sigma_{\text {Res }}$ in the cross-section of the structure after forming:

$$
\begin{aligned}
\sigma_{\text {Res }} & =\sigma^{1}+\sigma_{\text {Compr }}^{3}+\sigma_{\text {Bend }}^{3} \\
& =\sigma^{1}-\frac{S_{R}}{S_{R}+S_{T}} \sigma^{1}-M_{f x}\left(y-y_{G}\right) /\left(I_{R}^{G}+I_{T}^{G}\right)
\end{aligned}
$$


Fig. 3 shows the normal stresses in a cross-section of a stiffened panel formed using springback-forming. This stiffened panel is composed of a plate with a rectangular cross-section $\left(B=170 \mathrm{~mm}, e_{T}=2.4 \mathrm{~mm}\right)$ and a stiffener with a rectangular cross-section $\left(e_{R}=2.4 \mathrm{~mm}, h=17.5 \mathrm{~mm}\right)$. The material's Young's modulus of both parts is $70500 \mathrm{MPa}$ and the initial tensile stress applied $\sigma^{1}=218 \mathrm{MPa}$. The figure also shows stress levels in the structure

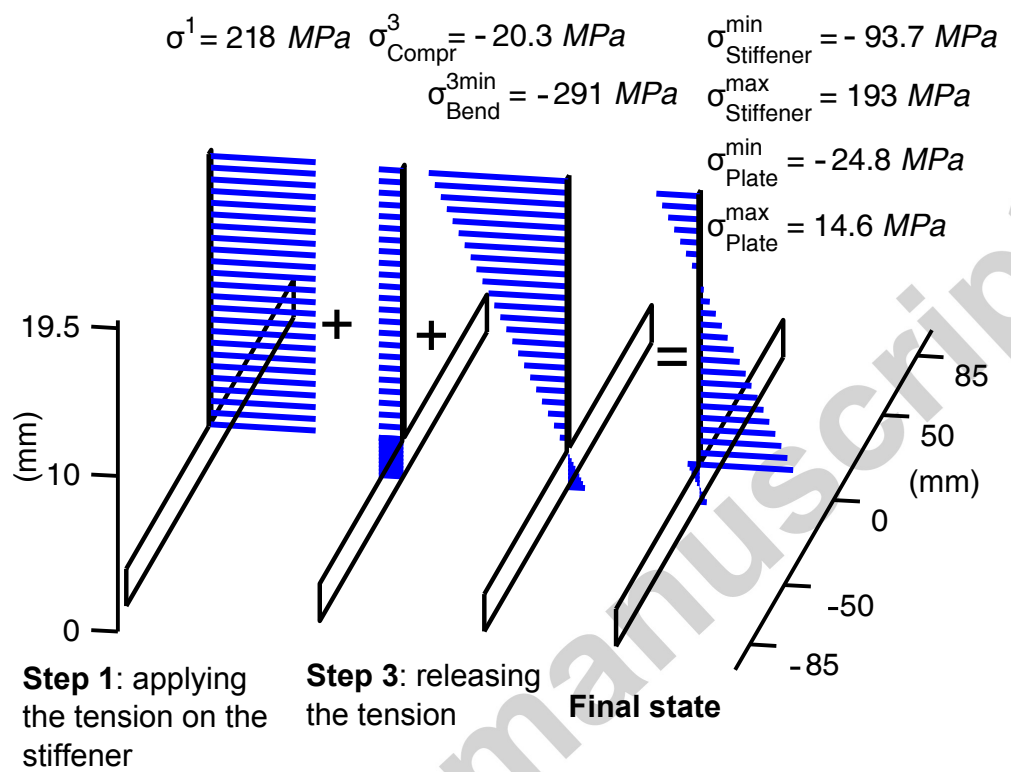

Fig. 3: Example of residual stresses in the structure after its forming by springbackforming.

at each step of the process. We note that the maximum residual stress (193 MPa), after forming, is located at the base of the stiffener (interface stiffener-plate). In this particular example, this value is smaller than the initial applied stress; however, it could by higher in certain cases. On the other hand, in the plate we note the existence of a typical bending stress profile because the cross-section centroid is located in the plate's thickness. Otherwise, when this centroid is in the stiffener, we could have only tensile or compressive stresses in the plate. In any case, we note that the plate's stress levels are smaller compared with the initial tensile stress. Furthermore, as we supposed that the plate is stress free in step 1, we observe a stress discontinuity at the interface stiffener-plate; hence, a shearing stress will occur 
at this interface.

In this article, we neglected the effects of the assembly process on springbackforming. However, some of these effects are already considered in the analytical analysis presented in this section. Indeed, using riveting or bolting as a joining process requires drilling holes and use of a flange or a parallel area to the plate. In any case, it means that the cross-section geometry of the plate or the stiffener or both of them has changed. These changes are considered through the bending moment formula, Eq. 1, and the longitudinal curvature radius expression Eq. 2. The stress distribution change in the structure will be partially considered by the residual stress equation, Eq. 9. However, in and nearby every cross-section where rivets or bolts are used, the stress distribution will slightly change and higher stresses values will concentrate near the drilled holes.

On the other hand, using welding as a joining process introduces, in the structure, distortions and a residual stress field that interacts with the pre-stress applied to the stiffener. These distortions and the distribution of residual stress are complex. They require a detailed study to analyze their effect on the process. Such a study is beyond the scope of this article and will be treated in another publication.

\section{Numerical simulation of springback-forming}

In this section, we present a finite element (FE) model developed in Abaqus (6.8) software environment to study the forming of a stiffened panel by springback-forming. With this numerical model, we were able to integrate the geometric and material non-linearities and also to obtain a more realistic distribution of residual stresses, particulary in the plate.

\subsection{Finite element model presentation}

The stiffened panel simulated, in this paragraph, has a cross-section similar to the example studied in the mechanical analysis Section 2.4. The parts length is $4 \mathrm{~m}$. Both are made from an isotropic aluminum alloy $6056 \mathrm{~T} 4 \mathrm{with}$ a Young's modulus $E=70500 \mathrm{MPa}$, a Poisson ratio $\nu=0.33$, and a flow curve extracted from Davoodi (2006) and presented in Fig. 4. The initial tensile stress applied to the stiffener is $\sigma^{1}=300 \mathrm{MPa}$.

Taking into account the pre-strain dependence of the Young's modulus would increase the quantitative accuracy of the model. However, it would not change the qualitative conclusions. As the biggest strain applied in all the 
simulations did not exceed $4 \%$ and to keep the model simple, we neglected this dependence.

We assumed that the material follow an elasto-plastic law with isotropic hardening behavior (Von Mises plasticity model). The springback calculation accuracy is highly influenced by Bauschinger effect, but only when the material undergoes a complicated cyclic deformation; which is note the case in springback-forming. In addition, no reverse yielding will occur in all the simulations carried out in this article. For these two reasons, we neglected the Bauschinger effect and the influence of the yields-locus of the material. Considering the longitudinal symmetry only half of the structure's length is

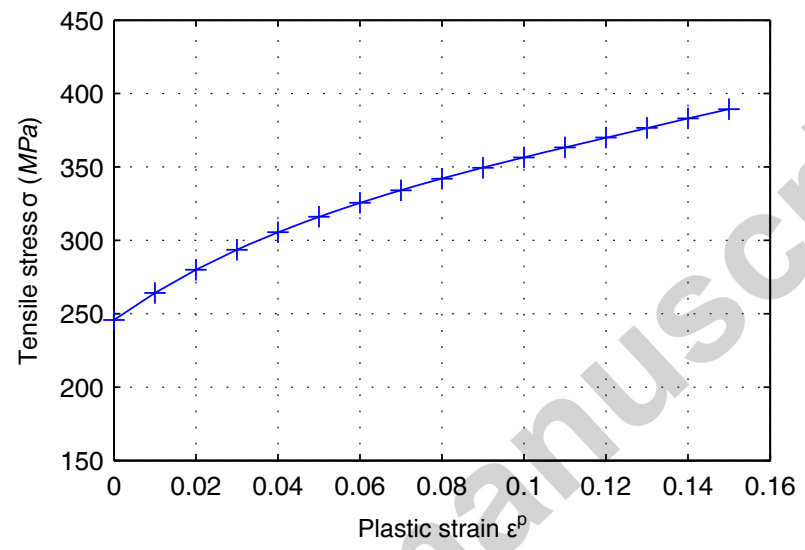

Fig. 4: Hardening curve of the aluminum alloy $6056 \mathrm{T4}$ at $20^{\circ} \mathrm{C}$ (Davoodi, 2006).

modeled. The geometrical non-linearities are taken into account. To mesh the structure, we used shell elements S4R (4 nodes and a reduced integration), issued from the elements library of Abaqus. In all the simulations carried in this work, the size of the elements is $\simeq 2 \mathrm{~mm} \times 2 \mathrm{~mm}$ in the stiffener and is $\simeq 4 \mathrm{~mm} \times 4 \mathrm{~mm}$ in the plate. Fig. 5 shows an example of such mesh.

The assembly stiffener-plate is modeled as rigid constraint between the edges in contact. The simulation consist of three static steps: (i) applying the tension on the stiffener, (ii) activating the rigid constraint, (iii) and finally releasing gradually the stiffener's tension. 


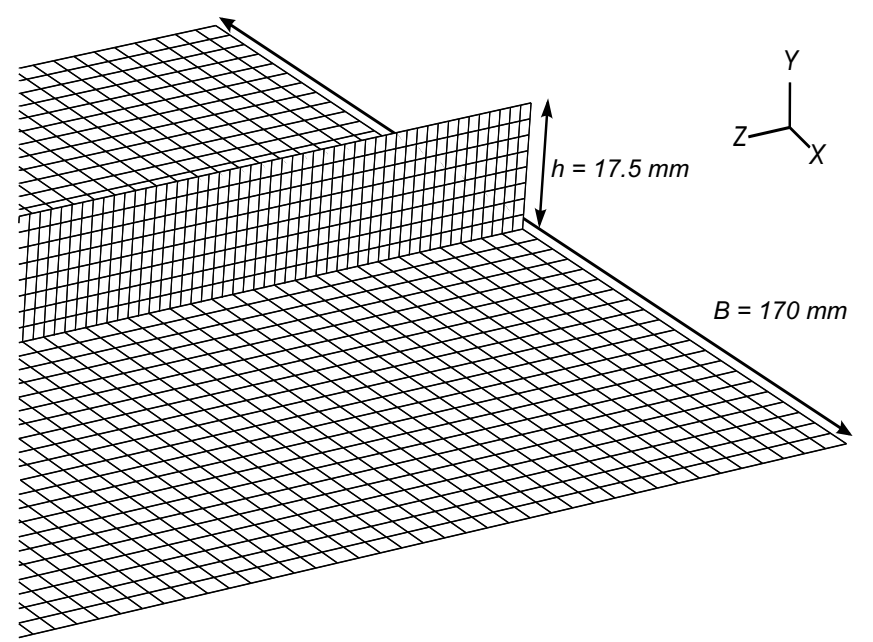

Fig. 5: Example of mesh used in the finite element model.

\subsection{Curvature characterization}

As we are interested in the curvature radius, we need a detailed analysis of this radius in every point of the panel. So, after forming, the plate is discretized into $m$ longitudinal fibers (Fig. 6.b) parallel to the stiffener. The position of a fiber $j(j=1, . . m)$ along the X-direction is noted $x_{j}$. Each fiber is discretized into $n$ points $M_{i=1, . . n}$. To calculate the curvature radius $R\left(x_{j}\right)$ of a fiber $j$, we fit a circular segment by minimising

$$
\Delta(R, O)=\sum_{i=1}^{n}\left(R-\left\|\overrightarrow{O M}_{i}\right\|\right)^{2}
$$

where $O$ is the circle's center, which is in the longitudinal symmetry plane $Z=0$ of the plate. Once we determine the couple $\left(R\left(x_{j}\right), O\left(x_{j}\right)\right)$, we define a radial error for the fiber $j$ (in $m m$ unit like a dimensional tolerance)

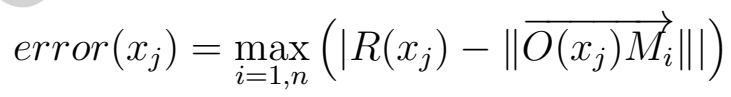

which indicates the degree of uniformity of the fiber's curvature (Fig. 6.a). So, for each panel we can plot the transverse variation of the curvature radius and the radial error versus the fibers' position $x_{j}$ (Fig. 6.b).

To have a global characteristic of the panel's curvature, we define the transverse dispersion

$$
\Delta R=R_{\max }-R_{\min }
$$




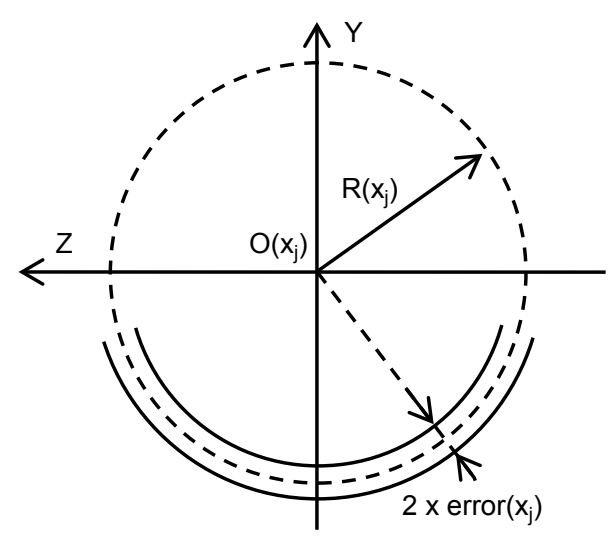

(a) Curvature characterization of a fiber in the plane $x=x_{j}$.

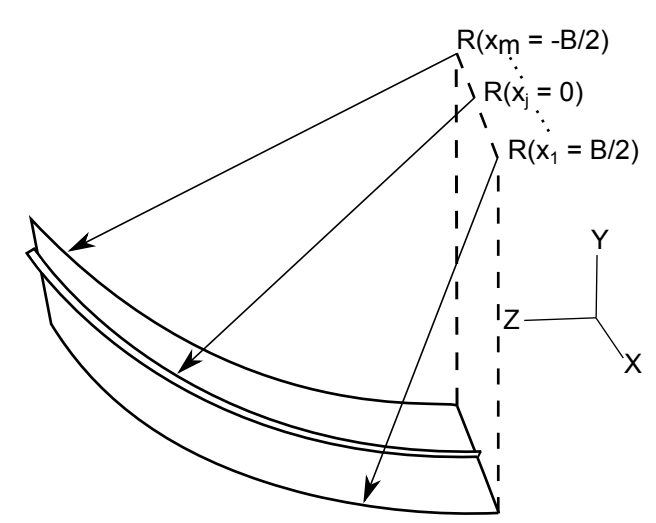

(b) Curvature radius of each longitudinal fiber.

Fig. 6: Characterization of the longitudinal curvature of a panel.

where $R_{\max }$ and $R_{\min }$ are the maximum and the minimum value of $R$. Thus, for a panel, we dispose of three characteristics: (i) the curvature radius of the plate's longitudinal center fiber (the one in contact with the stiffener), (ii) the transverse dispersion $\Delta R$, (iii) and the maximum of the radial errors.

\subsection{Deformation, curvature radius and residual stresses after forming}

We simulated the case defined in the model presentation Section 3.1. The deformation of the plate after forming is shown by displacement of its nodes in Fig. 7. Far from the plate's ends, the transverse displacement distribution is relatively uniform along its length, Fig. 7.a. However, the maximum amplitude is negligible $(\leq 0.014 \mathrm{~mm})$. On the other hand, no noticeable deflection of the plate's corners is observed in Fig. 7.b and Fig. 7.c.

Following the definitions of the previous section, Fig. 8 shows the detailed analysis of the panel's curvature radius. We note that the radial error, in all the panel, is inferior to $0.2 \mathrm{~mm}$, which indicates that the curvature radius is quit uniform in every sheet's fiber. Moreover, the transverse dispersion $\Delta R$ is inferior to $0.3 \mathrm{~mm}$, which indicates that the panel has a good uniformity of its curvature radius.

Fig. 9 shows the longitudinal residual stress distribution in the stiffener after forming (the other components of the Cauchy tensor stress are negligible). We see that the higher stresses are located near the interface stiffener-plate. 


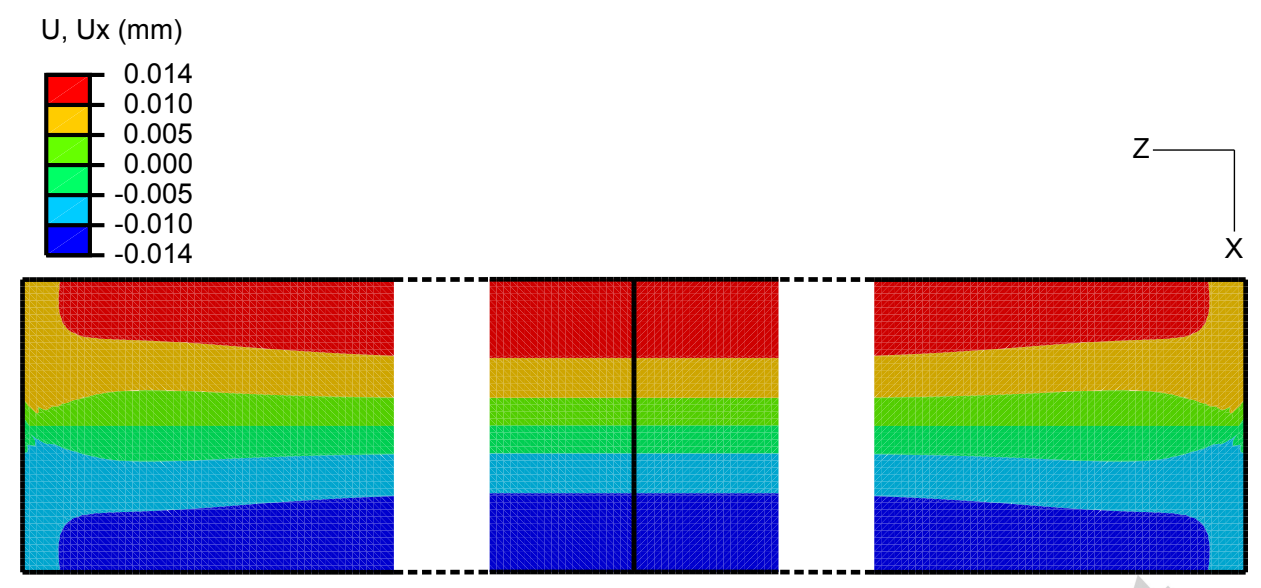

(a) Transverse displacement (X-direction) of the plate's nodes: $U x$.

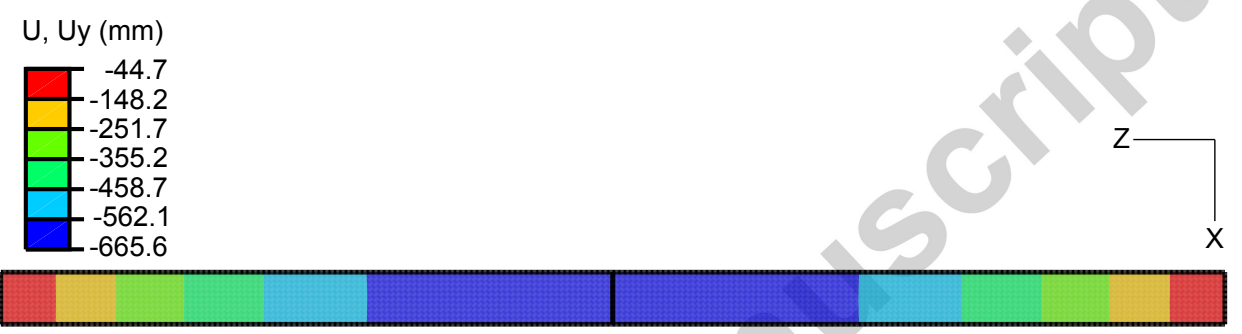

(b) Vertical displacement (Y-direction) of the plate's nodes: $U y$.

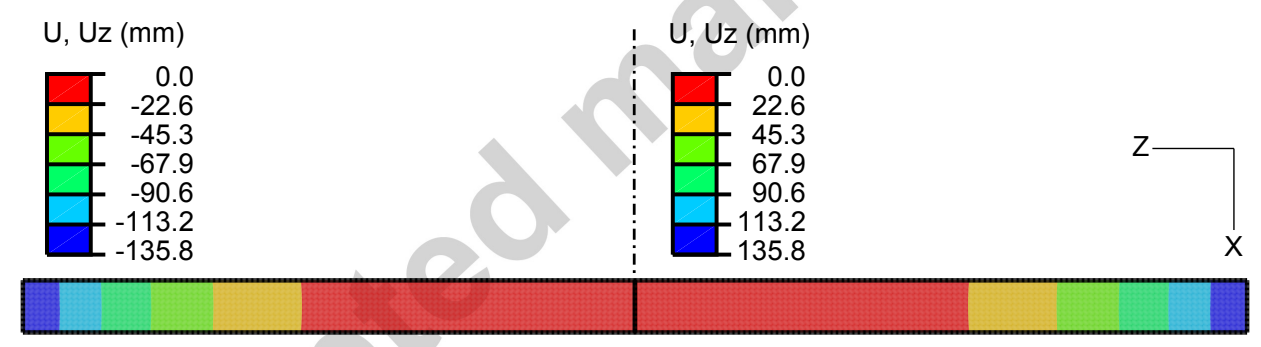

(c) Longitudinal displacement (Z-direction) of the plate's nodes: $U z$ (each half of the plate has its legend).

Fig. 7: Plate's nodes displacement after forming by springback-forming (plate: $4 \mathrm{~m} \times$ $170 \mathrm{~mm} \times 2.4 \mathrm{~mm}$, stiffener: $4 \mathrm{~m} \times 17.5 \mathrm{~mm} \times 2.4 \mathrm{~mm}$, initial applied stress $\sigma^{1}=300 \mathrm{MPa}$ ).

To illustrate the distribution of the stresses in a stiffener's cross-section, Fig. 10.a shows the principal stresses of the stiffener's middle section (plane $Z=0$ ) obtained using the FE model. To compare it to the analytical model's results, Fig. 10.b shows the stresses obtained by the analytical model (Section 


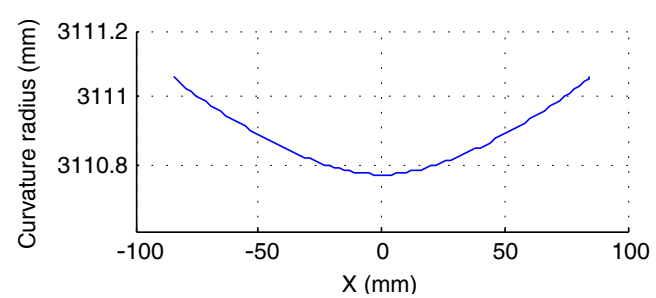

(a) Transverse variation of the longitudinal curvature radius.

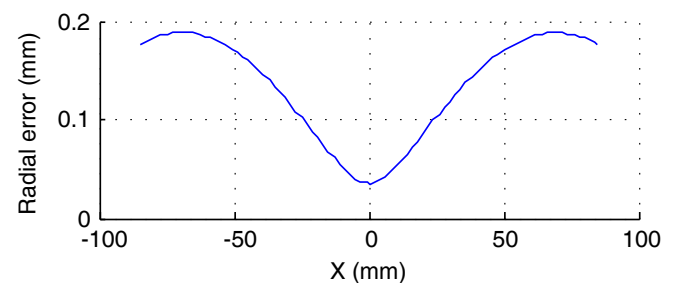

(b) Transverse variation of the radial error.

Fig. 8: Curvature characterization of a panel after forming by springback-forming (plate: $4 \mathrm{~m} \times 170 \mathrm{~mm} \times 2.4 \mathrm{~mm}$, stiffener: $4 \mathrm{~m} \times 17.5 \mathrm{~mm} \times 2.4 \mathrm{~mm}$, initial applied stress $\sigma^{1}=$ $300 \mathrm{MPa})$.

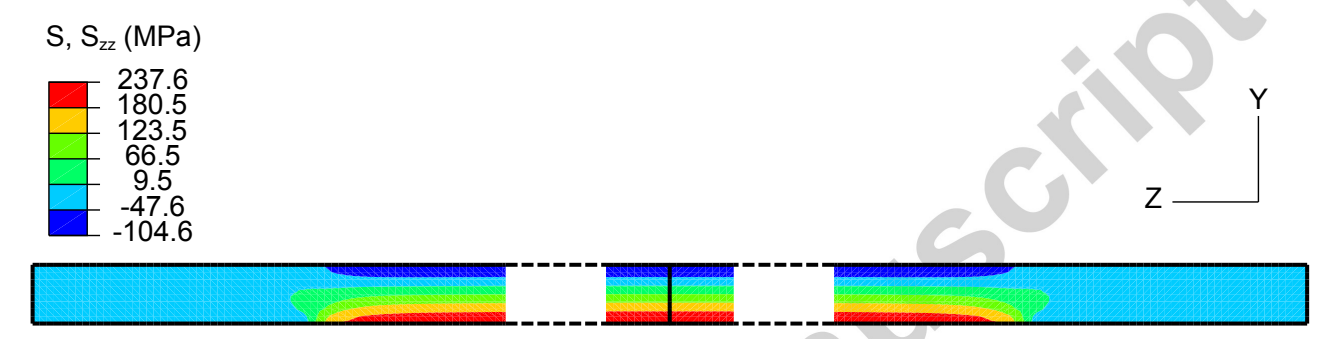

Fig. 9: Longitudinal residual stress in the stiffener after forming.

2.4) as well as extreme stress values in the stiffener and in the plate. We note that there is a good qualitative correlation between the results.

The principal stresses of the plate's middle section $(Z=0)$ obtained using the FE model are shown in Fig. 11. We note that, as in the stiffener, the longitudinal stresses are dominant. Though the analytical model predicts a constant stress along the X-direction in the plate's cross-section, as shown by Fig. 10.b, the FE model shows a variation $\leq 16 \mathrm{MPa}$ of this stress. However, we note that they are of the same nature as the analytical stresses (compressive stresses in sheet's superior surface and tensile stresses on the other surface). To evaluate the stress field in the plate, Fig. 12 shows the distribution of the Von Mises stress in both its superior and inferior surfaces. We observe that far from a limited zone near the plate's ends, in contact with the stiffener, the stress level does not exceed $54 \mathrm{MPa}$. As shown in Fig. 9 and Fig. 12, we note also that far from the plate's ends, the spatial distribution of residual stresses is relatively uniform along the length of the structure. 


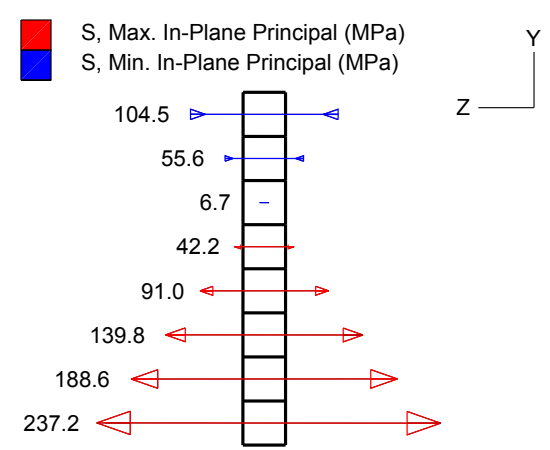

(a) Numerical principal stresses in the stiffener's middle cross-section.

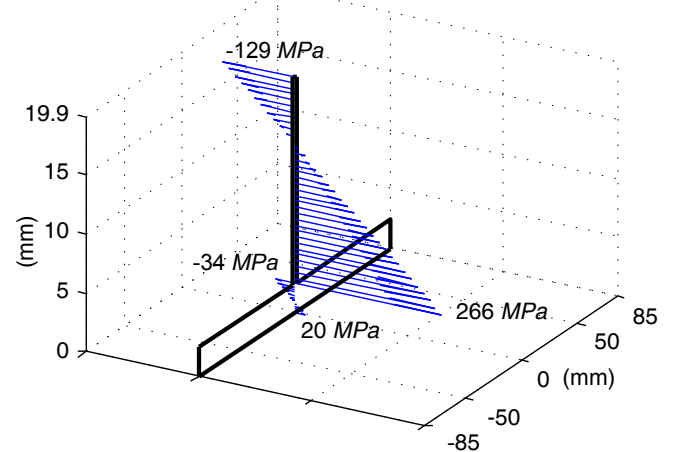

(b) Longitudinal residual stresses in the structure middle cross-section obtained by the analytical model.

Fig. 10: Principal stresses in a structure's middle cross-section after forming.

S, Max. In-Plane Principal (MPa
S, Min. In-Plane Principal (MPa)

(a) Numerical principal stresses in the superior surface of the plate, in contact with the stiffener.

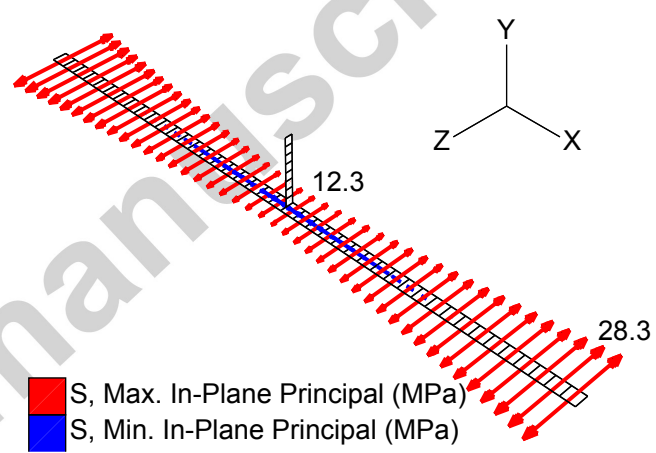

(b) Numerical principal stresses in the inferior surface of the plate.

Fig. 11: Principal stresses in the central transverse section of the plate after forming.

\section{Parametrical analysis and an experimental test of springback- forming}

We consider the structure geometry described in Section 3.3 as a reference configuration. Using the FE model, we study in this section, the curvature radius variation with respect to one mechanical parameter: the initial stiffener's tensile stress $\sigma^{1}$; and with respect to three geometrical parameters: 


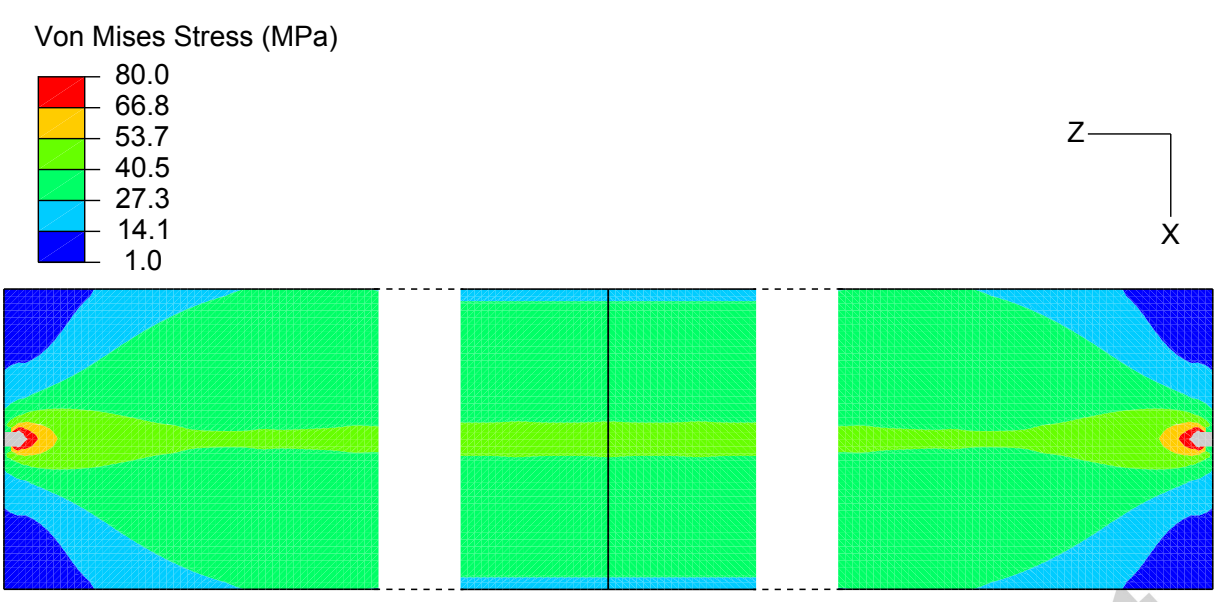

(a) Plate's superior surface in contact with the stiffener.

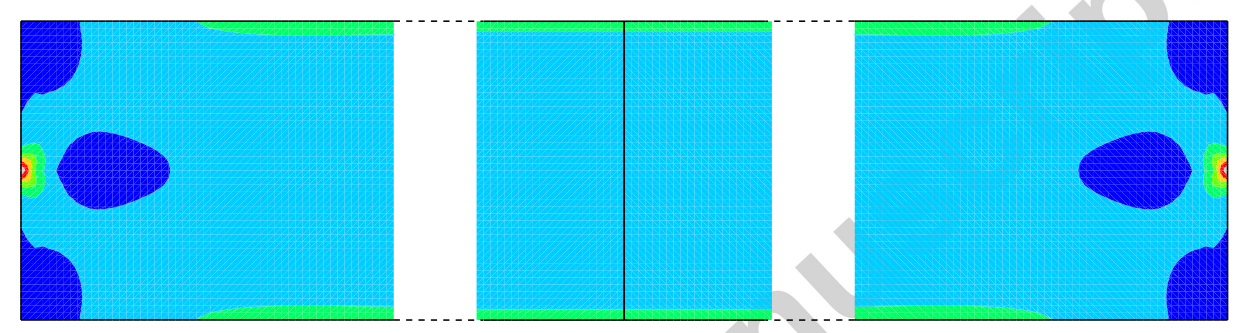

(b) Plate's inferior surface.

Fig. 12: Von Mises stresses in the plate after its forming by springback-forming.

stiffener's height $h$, plate's width $B$, and structure's length $L$. For each parameter variation, numerical to analytical results are compared. In addition, we present the results of three experimental tests of springback-forming.

\subsection{Initial stiffener's tension effect}

To analyze the effect of the initial stiffener's tension, different pre-load values, ranging from $6 k N$ to $12.1 k N$, were applied on the stiffener. These pre-loads induce stresses below and above the material's yield point (Fig. 4). Some stress values with the corresponding applied forces and longitudinal strains are given in Table 1.

Fig. 13 gives the variation of the curvature radius of the plate's longitudinal center fiber, obtained by the numerical and the analytical model, with respect to the stiffener's initial tensile stress. In all the simulations, the ra- 


\begin{tabular}{|l|c|c|c|c|c|}
\hline Tensile stress $\sigma^{1}(\mathrm{MPa})$ & 142 & 219 & 246 & 254 & 300 \\
\hline Elastic strain $\epsilon_{z z}^{e}(\%)$ & 0.2 & 0.31 & 0.35 & 0.36 & 0.43 \\
\hline Plastic strain $\epsilon_{z z}^{p}(\%)$ & 0 & 0 & 0.02 & 0.45 & 3.54 \\
\hline Applied force $(k N)$ & 6 & 9.2 & 10.3 & 10.6 & 12.1 \\
\hline
\end{tabular}

Table 1: Initial loads and corresponding tensile stresses applied to the stiffener $(4 \mathrm{~m} \times$ $17.5 \mathrm{~mm} \times 2.4 \mathrm{~mm})$.

dial error is inferior to $0.2 \mathrm{~mm}$, which indicates that the curvature radius is uniform in every plate's fiber. Additionally, the transverse dispersion $\Delta R$ is inferior to $0.36 \mathrm{~mm}$, which indicates that the panel, in every case, have a good uniformity of its curvature radius. On the other hand, the results of both models are very similar, as the difference is inferior to $0.4 \%$. This small

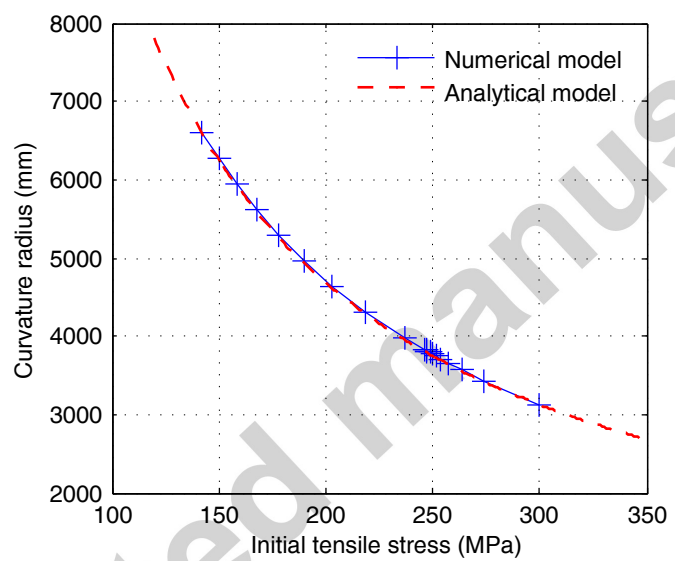

Fig. 13: Variation of the plate's curvature radius with respect to the initial tensile stress applied to the stiffener.

difference shows that, for this structure, the analytical model gives a good estimation of the curvature radius.

\subsection{Stiffener height's effect}

For this study, the stiffener's height varies from 6 to $250 \mathrm{~mm}$; but, the other parameters are the ones of the reference configuration $\left(e_{R}=e_{T}=\right.$ $2.4 \mathrm{~mm}, B=170 \mathrm{~mm}, L=4000 \mathrm{~mm}$, and $\sigma^{1}=300 \mathrm{MPa}$ ). 
The results of the simulations are synthesized in Fig. 14. The radial error, in all these simulations, is inferior to $0.4 \mathrm{~mm}$, which indicates the uniformity of the curvature radius of all the plate's longitudinal fibers. We note, additionally, in Fig. 14.a, that both curves (analytical and numerical) have a minimum value of the plates' curvature radius when $h\left(R_{\min }\right)=7 \mathrm{~mm}$. We note also that the curvature radius increases significantly with the increasing of the stiffener's height (more than $1.3 \mathrm{~m}$ for every $10 \mathrm{~mm}$ increase of $h$ ). This result could be a limitation of the use of springback-forming for stiffened panels with high height stiffeners.

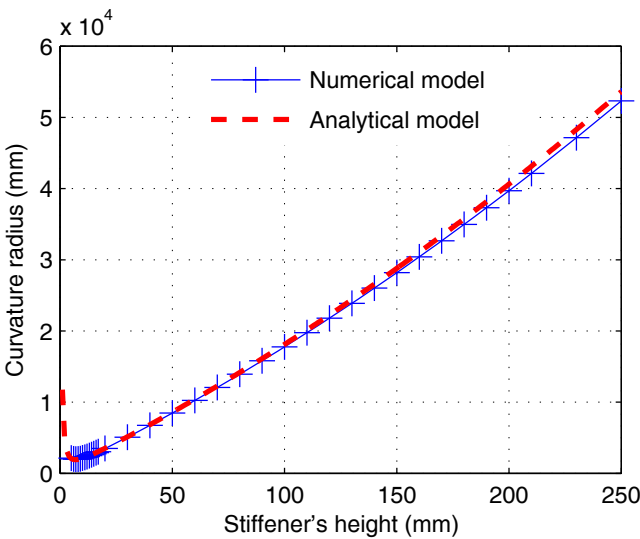

(a) Variation of the curvature radius of the plate's longitudinal center fiber with respect to the stiffener's height.

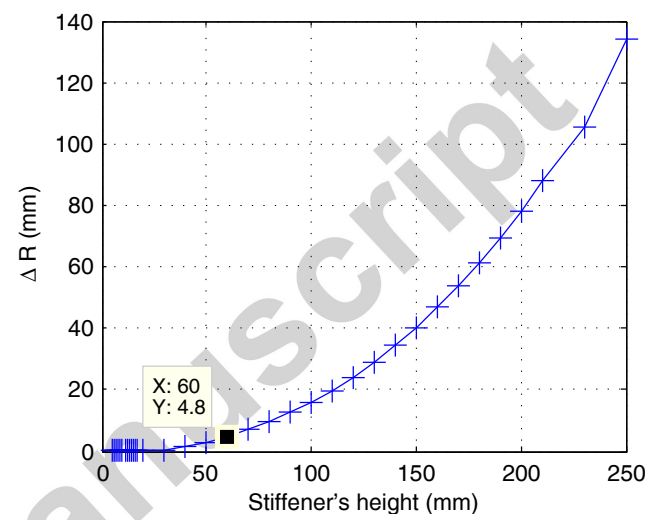

(b) Variation of the curvature radius's transverse dispersion $\left(\Delta R=R_{\max }-R_{\min }\right)$ in the plate with respect to the stiffener's height.

Fig. 14: Stiffener's height effect on the plate's curvature radius after forming.

Moreover, we observe a good agreement between numerical and analytical results, as the difference is inferior to 2.3\%. However, as shown in Fig. 14.b, the transverse dispersion of the curvature radius, which is absent in the analytical model, increases with the stiffener's height. Relatively to the curvature radius this dispersion is inferior to $0.3 \%$. But this dispersion could be unacceptable during the assembly of the stiffened panel with an other structure. In case of an imposed tolerance on the curvature radius uniformity, the curve presented in Fig. 14.b. would be used to determine the maximum value of $h$, for which with springback-forming that tolerance will be respected. 


\subsection{Plate width's effect}

The capability of the springback-forming to impose a uniform curvature radius on a given plate's width is important. Indeed, it has a direct impact on how many stiffeners should be used to form a larger panel, and expect to have a uniform curvature radius. To study the effect of the plate's width on the process, we fixed the rest of the parameters to those of the reference configuration $\left(e_{R}=e_{T}=2.4 \mathrm{~mm}, h=17.5 \mathrm{~mm}, L=4000 \mathrm{~mm}\right.$, and $\sigma^{1}=300 \mathrm{MPa}$ ) and we varied $B$ from $10 \mathrm{~mm}$ to $1800 \mathrm{~mm}$.

The variation of the curvature radius (of the plate's longitudinal center fiber) versus the plate's width, obtained with both models (numerical and analytical), is given in Fig. 15.a. We note a relatively good agreement between the numerical and analytical results, as the difference is inferior to $2.3 \%$.

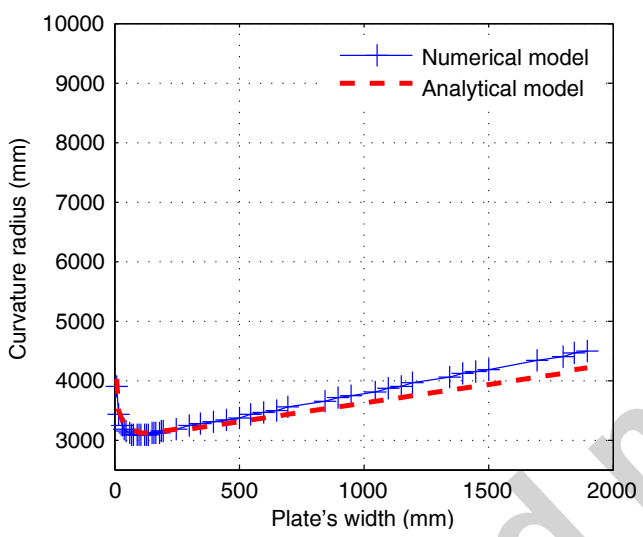

(a) Variation of the curvature radius of the plate's longitudinal central fiber with respect to the plate's width.
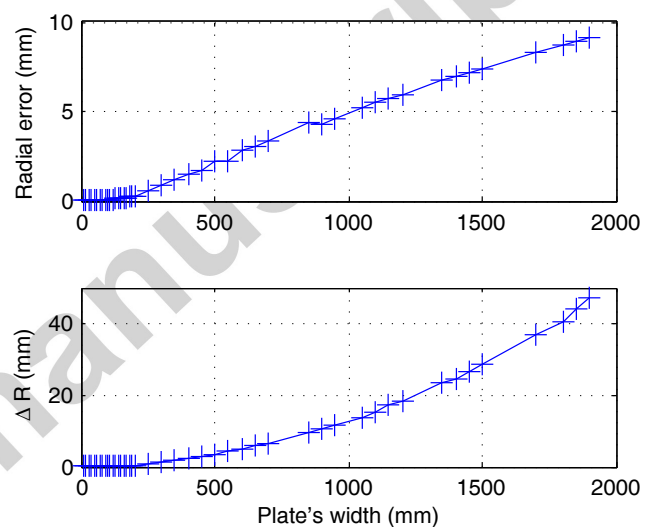

(b) Variation of curvature radius's transverse dispersion $\left(\Delta R=R_{\max }-R_{\min }\right)$ and the radial error in the plate with respect to the plate's width.

Fig. 15: Plate's width effect on its curvature radius after forming.

The same figure shows the existence of a minimum value around $B\left(R_{\min }\right)=$ $110 \mathrm{~mm}$. In Fig. 15.b, we note that the radial error and the transverse dispersion increase with the plate's width. This increase indicates a decrease of both the longitudinal and transversal uniformity of the curvature radius. We observe the same tendencies as with stiffener's height. However, the width's plate has a weaker effect (than the stiffener's height) on the curvature 


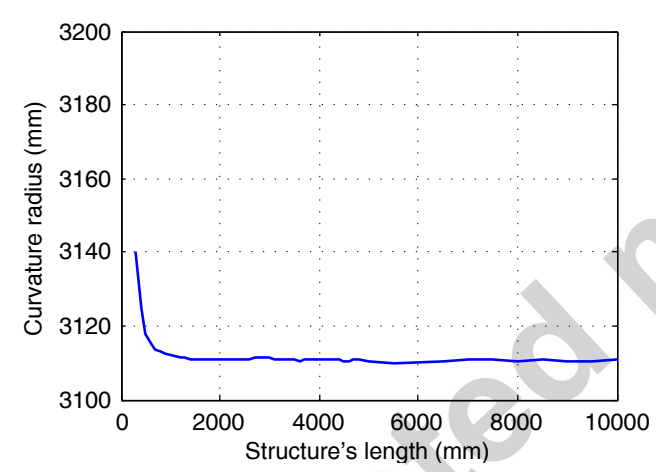

(a) Variation of the curvature radius of the plate's central fiber with respect to the structure's length.

To study the effect of the structure's length on the curvature radius, we considered the reference configuration dimensions $\left(h=17.5 \mathrm{~mm}, e_{R}=e_{T}=\right.$ $2.4 \mathrm{~mm}, B=170 \mathrm{~mm}$, and $\sigma^{1}=300 \mathrm{MPa}$ ) and we changed the length from $0.3 \mathrm{~m}$ to $10 \mathrm{~m}$ with a step of $100 \mathrm{~mm}$ up to $5 \mathrm{~m}$ and a step of $500 \mathrm{~mm}$ up to $10 \mathrm{~m}$.

Fig. 16.a shows the variation of the curvature radius of the plate's longitudinal center fiber for different structure's length. In all the simulations the

Fig. 16: Structure's length effect on the plate's curvature radius after forming.

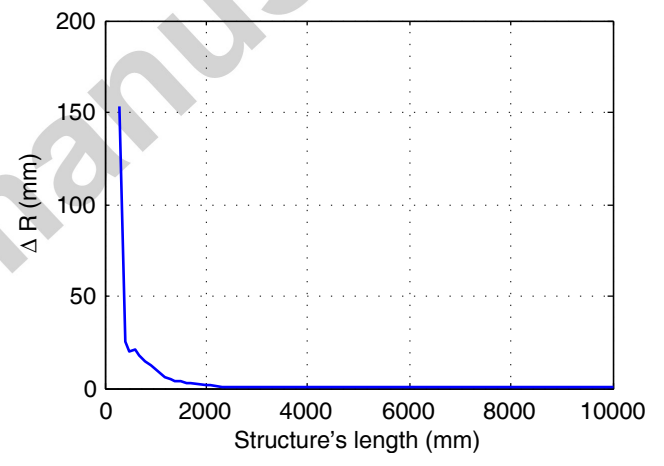

(b) Variation of the curvature radius's transverse dispersion $\left(\Delta R=R_{\max }-R_{\min }\right)$ in the plate with respect to the structure's length.

radial error is inferior to $0.3 \mathrm{~mm}$, which indicates that the curvature radius is uniform in all plate's longitudinal fibers. We note that the value of the curvature radius is quasi-constant for all the lengths, as the variation is inferior to $40 \mathrm{~mm}$. However, the transverse dispersion is greater for small values 
of the structure's length (up to $150 \mathrm{~mm}$ ), Fig. 16.b.

We also note, in Fig. 16, that when the structure is long enough (length $\geq 2 \mathrm{~m}$ ) compared with structure's cross-section dimensions, the curvature radius value becomes constant $( \pm 0.5 \mathrm{~mm})$ and the transverse dispersion becomes small $(\leq 1.4 \mathrm{~mm})$. In other words, the curvature radius becomes independent of the initial structure's length.

\subsection{An experimental test of springback-forming}

To test the feasibility of springback-forming experimentally, we conducted three forming tests. The objective of these tests is: - to demonstrate the capability of the process to bend a single-curved structure; - to show the repeatability of the results; - to test the sensibility of the process to the initial stiffener's tension; - and to compare the curvature radius obtained numerically and experimentally.

The geometry and the material of the structure, in the three tests, is the same as in the mechanical analysis Section 2.4. As we showed that the results of bending using springback-forming are independent of the structure's length, we chose the plates length $355 \mathrm{~mm}$ and the stiffeners length $541 \mathrm{~mm}$. The additional length of the stiffeners is necessary to allow the use of grips to apply tension during the first phase of the forming process. The thickness of all parts is $2.4 \mathrm{~mm}$ and the plates width is $170 \mathrm{~mm}$. The rest of the characteristics of the tests is given in Table 2 .

\begin{tabular}{|l|c|c|c|}
\cline { 2 - 4 } \multicolumn{1}{c|}{} & Test 1 & Test 2 & Test 3 \\
\hline Stiffeners height $(\mathrm{mm})$ & 17.5 & 17.6 & 17.5 \\
\hline Initial tensile stress $\sigma^{1}(\mathrm{MPa})$ & 214.2 & 202.2 & 155.3 \\
\hline
\end{tabular}

Table 2: Stiffeners height and the initial tensile stress applied to them in the three experimental tests of springback-forming.

As a joining process, welding is more challenging than riveting or bolting to integrate in springback-forming. Nevertheless, it helps reducing the structure mass by simplifying the interface between the stiffeners and the plate. It is also easy to automate and easily adaptable to complex geometries. For these reasons, we used laser beam welding as a joining process in the experiments. We used a YAG welding source to form a tee joint between the stiffener and the plate without a filler material. The laser beam was focused in the plate's 
inferior surface, perpendicular to the plate, positioned above and in the opposite side of the stiffener; and had a diameter of $0.2 \mathrm{~mm}$, a travelling speed of $0.6 \mathrm{~m} /$ minute and a power of $1700 \mathrm{~W}$. This welding configuration was used to keep the longitudinal symmetry of the structure. As a shielding gas, we used argon with a flow rate of 20 liter/minute. With the imposed welding parameters, we obtained a welding bead with little porosity, a uniform width and no visible fissures. In addition, the fusion zone included a part from the stiffener throughout its length.

To measure the geometrical shape of the structure after forming, we used a coordinate measuring machine with a sphere as probe. As we are interested in the longitudinal curvature, we measured the points' coordinates of 17 fibers equally spaced along the width of the plate and parallel to the stiffener. Each fiber was composed of 70 points equally spaced. The precision of coordinate measures, using a sphere as probe, was quite good: $10^{-3} \mathrm{~mm}$ for $\mathrm{X}$-coordinates, $10^{-2} \mathrm{~mm}$ for Y-coordinates and $10^{-1} \mathrm{~mm}$ for Z-coordinates. A result of this measuring procedure applied to Test 2 is given in Fig.17.

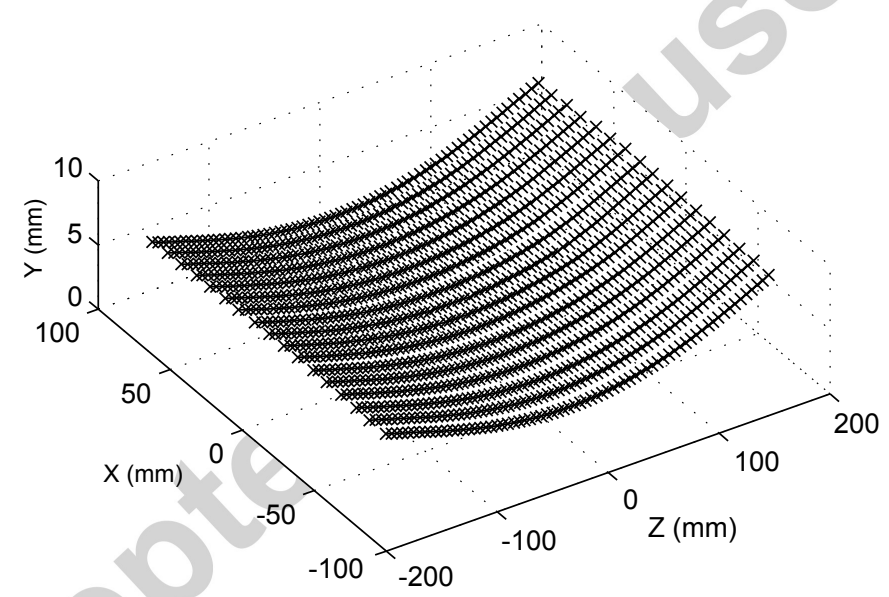

Fig. 17: Example of points, measured using the coordinate measuring machine, of a panel formed by springback-forming. (Test 2: plate: $355 \mathrm{~mm} \times 170 \mathrm{~mm} \times 2.4 \mathrm{~mm}$, stiffener: $544 \mathrm{~mm} \times 17.6 \mathrm{~mm} \times 2.4 \mathrm{~mm}$, initial applied stress $\sigma^{1}=202.2 \mathrm{MPa}$ ).

From these measures, and following the curvature characterization procedure of Section 3.2, Fig. 18 shows the transverse variation of the curvature radius of the three tests' plates. The maximum radial error is $2 \mathrm{~mm}$, which shows a good uniformity of the curvature radius of the plates' longitudinal fibers. 
These experimental curves demonstrates clearly the feasibility of the process and that with springback-forming we can obtain a single-curved panel. It shows also that the results are repeatable. Indeed, if we consider Test 1 and Test 2, we observe that the geometrical parameters are practically identical and that the initial applied tensile stresses are close. Additionally, we note that the curvature radius decreases when we increase the initial tensile stress applied to the stiffener. This is coherent with the conclusion of the analytical and numerical model (Section 4.1).

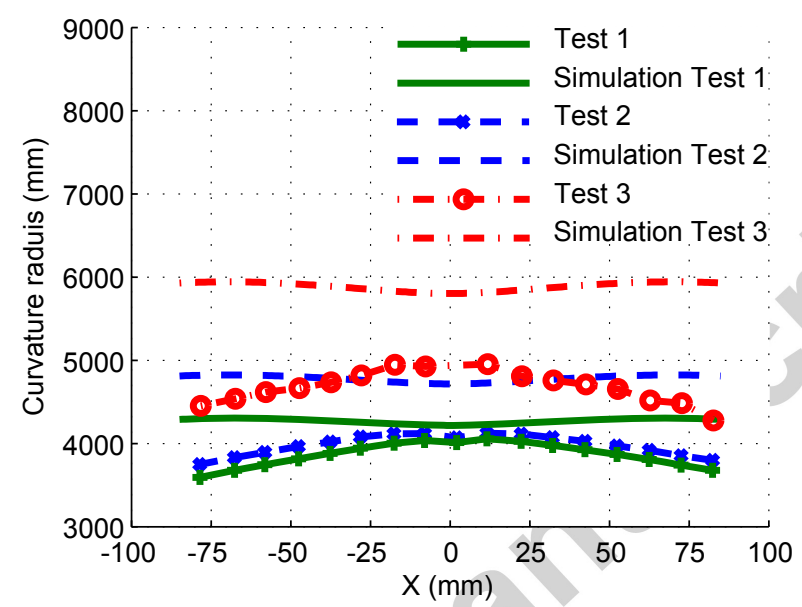

Fig. 18: Experimental and numerical transverse variation of longitudinal curvature radius of the three tests' panels, after forming by springback-forming (Test 1: $\sigma^{1}=214.2 \mathrm{MPa}$, Test 2: $\sigma^{1}=202.2 \mathrm{MPa}$, Test 3: $\sigma^{1}=155.3 \mathrm{MPa}$ ).

On the other hand, using the developed numerical model, we simulated the three tests. Fig. 18 shows the transverse variation of the curvature radius of the panels. The radial error of the three simulations' results is inferior to $0.2 \mathrm{~mm}$. To compare numerical and experimental results, Tab. 3 summarizes the curvature radius of the plate's longitudinal center fiber and the transverse dispersion of each test. We note that the numerical model overestimates the curvature radius and that the difference, between the two values, increases with the decreasing of the initial applied stress (from $4.9 \%$ in Test 1 to $15 \%$ in Test 3). As for the transverse dispersion, the numerical model underestimates it. This gap between the numerical and experimental result is because we ignored the effect of the assembly process. However, we are aware that welding introduces distortions and residual stresses that interact 
with springback-forming process. To have a better quantitative estimation of the results and more insight into theses interactions, a model integrating the simulation of laser beam welding should be developed.

\begin{tabular}{|l|c|c|c|}
\cline { 2 - 4 } \multicolumn{1}{c|}{} & Test 1 & Test 2 & Test 3 \\
\hline Experimental curvature radius, $R_{e}(\mathrm{~mm})$ & 4010 & 4069 & 4927 \\
\hline Numerical curvature radius, $R_{n}(\mathrm{~mm})$ & 4218 & 4717 & 5810 \\
\hline Difference between $R_{e}$ and $R_{n}$ in percentage(\%) & 4.9 & 13.7 & 15.1 \\
\hline Experimental transverse dispersion $(\mathrm{mm})$ & 462 & 377 & 676 \\
\hline Numerical transverse dispersion $(\mathrm{mm})$ & 86 & 108 & 141 \\
\hline
\end{tabular}

Table 3: Comparison of curvature radius of the plate's longitudinal center fiber and the transverse dispersion obtained numerically and experimentally from the three tests of springback-forming.

\section{Conclusion}

In this article, we have presented a forming process dedicated to stiffened panels that we named springback-forming. It is based on the idea to apply a tension on the stiffener before assembling it with the panel in a flat configuration. The forming of the structure is achieved after releasing the stiffener's tension. It has the merit of its reduced machines costs (as it uses mainly a tension tool) and its adaptability to all panel sizes. By analyzing the process steps, in the case of a plate with one stiffener, we showed that it is possible to obtain a single-curved panel using the springback energy stored in the stiffener. The concept of pre-stressing the stiffeners before the assembly could be used in forming more complex panels.

To study the capabilities and the limitations of the process, an analytical and a numerical model were developed. The analytical model is based on Euler-Bernoulli beam theory. From this model we conclude that: - the more the material is flexible, the smaller is the achievable curvature radius; - the residual stresses in the structure are mainly longitudinal and the maximum value is around the initial applied tensile stress. We also showed the existence of minimum values of the curvature radius when analyzing its variation with each geometrical parameter.

To have a more general tool to analyze the process, we have developed a numerical simulation based on finite element method. To characterize the 
panel's curvature after forming, we have defined three parameters: a curvature radius of each longitudinal fiber of the plate, a radial error, and a transverse dispersion evaluating the uniformity of the curvature in the entire panel. The results of the numerical model, obtained through a parametrical study, are in good agreement with those of the analytical one. This good agreement makes the analytical model a quick tool to evaluate the effect of the parameters on the process. On the other hand, the numerical model allows the quantification of the uniformity of the panel's curvature radius. According to the numerical simulations of the studied case, we conclude that with the same initial applied force and above a minimum length $(2 \mathrm{~m}$ for the reference configuration), the curvature radius becomes independent of the structure's length. In contrast, the stiffener's height has a strong effect on the process. Indeed, the curvature radius, increases from $2 m$ to more than $10 \mathrm{~m}$ when the height changes from $17.5 \mathrm{~mm}$ to $60 \mathrm{~mm}$. The stiffener height could be a limitation to the minimum curvature radii achievable by the process. As for the plate's width, it has a weaker effect than the stiffener's height: the variation of the curvature radius is less than $2 m$ for a width variation of $1.8 \mathrm{~m}$. These conclusions are valid only for the considered structure. For other geometries, to define the achievable curvature radii and their uniformity, similar curves to those presented in this article, should be plotted. The experimental tests, carried out in this study, demonstrated that springback-forming is capable of bending a single-curved panel and that its sensitivity to initial tensile stress agrees qualitatively with the analytical and the numerical model predictions. Hence, the process could be considered as a viable alternative when choosing the most suitable process for a specific stiffened panel. However, quantitatively the numerical model overestimates the produced curvature radius and underestimates the transverse dispersion of this curvature radius. These differences are caused by laser beam welding used as joining process. More experiments together with the integration in the numerical model, of the distortions and the residual stress field introduced by the joining process, should give more insight into its effect on springbackforming.

\section{References}

Brewer, H., 1989. Age forming integrally stiffened, aluminum aerospace structures in an autoclave, in: Aircraft Design and Operations Meetings. American Institute of Aeronautics and Astronautics. doi:10.2514/6.1989-2087. 
575

576

577

Davoodi, B., 2006. Etude du comportement quasi-statique et dynamique des matériaux métalliques à haute température-simulation numérique du formage à chaud. Ph.D. thesis. INSA de Rennes.

Gariepy, A., 2012. Finite element modelling of shot peening and peen forming processes and characterisation of peened AA2024-T351 aluminium alloy. Ph.D. thesis. Ecole Polytechnique de Montreal.

Holman, M.C., 1989. Autoclave age forming large aluminum aircraft panels. Journal of Mechanical Working Technology 20, pp. 477-488. doi:10.1016/0378-3804(89)90055-7.

Li, K., 1981. Using stress peen-forming process for integrally stiffened wing panels, in: 1st International Conference on Shot Peening, Paris, France. pp. $555-564$.

Lin, J., Hoa, K.C., Dean, T.A., 2006. An integrated process for modelling of precipitation hardening and springback in creep age-forming. International Journal of Machine Tools and Manufacture 46, pp. 1266-1270. doi:10.1016/j.ijmachtools.2006.01.026.

Megson, T., 2010. Introduction to Aircraft Structural Analysis (Elsevier Aerospace Engineering). Butterworth-Heinemann.

Meyer, R., Reccius, H., Schulein, R., 1987. Shot peen-forming of nc-machined parts with integrated stringers using large balls, in: 3rd International Conferences on Shot Peening, Garmisch-Partenkirchen, Germany. pp. 327-334.

NASA-CR-124075, 1973. Isogrid design handbook. Technical Report. McDonnel Douglas Astronautics Company.

Pettit, R.G., Wang, J.J., Toh, C., 2000. Validated feasibility study of integrally stiffened metallic fuselage panels for reducing manufacturing costs. Technical Report NASA/CR-2000-209342. National Aeronautics and Space Administration.

Takafumi, A., Shirou, K., Takahiro, N., Hiroyuki, T., Masakazu, S., 2004. Age forming technology for aircraft wing skin. Materials Forum 28, pp. 202-207. 
Toros, S., Ozturk, F., Kacar, I., 2008. Review of warm forming of aluminummagnesium alloys. Journal of Materials Processing Technology 207, pp. 1-12. doi:10.1016/j.jmatprotec.2008.03.057.

Wang, T., Platts, M., 2002. A computer-aided blank design method for the peen forming process. Journal of Materials Processing Technology 122, pp. 374 - 380. doi:10.1016/S0924-0136(02)00049-3.

Yan, Y., Wan, M., Wang, H.B., 2009. FEM equivalent model for press bend forming of aircraft integral panel. Transactions of Nonferrous Metals Society of China 19, pp. 414 - 421. doi:10.1016/S1003-6326(08)60288-5. 


\section{List of Figures}

1 The three steps of springback-forming. . . . . . . . . . . . 5

(a) Step 1: applying a tension on the stiffener. . . . . . . 5

(b) Step 2: maintaining the stiffener's tension while assembling it with the plate. . . . . . . . . . . . 5 5

(c) Step 3: releasing the tension. . . . . . . . . . 5

2 Geometrical parameters of the assembly's cross-section (stiffener and plate). . . . . . . . . . . . . . . 6

3 Example of residual stresses in the structure after its forming by springback-forming. . . . . . . . . . . . . . . . 10

4 Hardening curve of the aluminum alloy $6056 \mathrm{~T} 4$ at $20^{\circ} \mathrm{C}$ (Davoodi, 2006). . . . . . . . . . . . . . . . . 12

5 Example of mesh used in the finite element model. . . . . 13

6 Characterization of the longitudinal curvature of a panel. . . . 14

(a) Curvature characterization of a fiber in the plane $x=x_{j} .14$

(b) Curvature radius of each longitudinal fiber. . . . . . . 14

$7 \quad$ Plate's nodes displacement after forming by springback-forming (plate: $4 \mathrm{~m} \times 170 \mathrm{~mm} \times 2.4 \mathrm{~mm}$, stiffener: $4 \mathrm{~m} \times 17.5 \mathrm{~mm} \times$ $2.4 \mathrm{~mm}$, initial applied stress $\sigma^{1}=300 \mathrm{MPa}$ ). . . . . . . . . 15

(a) Transverse displacement (X-direction) of the plate's nodes: Ux. . . . . . . . . . . . . 15

(b) Vertical displacement (Y-direction) of the plate's nodes: Uy. . . . . . . . . . . . . . . . 15

(c) Longitudinal displacement (Z-direction) of the plate's nodes: $U z$ (each half of the plate has its legend). . . . . 15

8 Curvature characterization of a panel after forming by springbackforming (plate: $4 \mathrm{~m} \times 170 \mathrm{~mm} \times 2.4 \mathrm{~mm}$, stiffener: $4 \mathrm{~m} \times$ $17.5 \mathrm{~mm} \times 2.4 \mathrm{~mm}$, initial applied stress $\left.\sigma^{1}=300 \mathrm{MPa}\right)$. . . 16

(a) Transverse variation of the longitudinal curvature radius. 16

(b) Transverse variation of the radial error. . . . . . . . . 16

9 Longitudinal residual stress in the stiffener after forming. . . . 16

10 Principal stresses in a structure's middle cross-section after forming. . . . . . . . . . . . . . . . . . . . . 17

(a) Numerical principal stresses in the stiffener's middle crosssection. . . . . . . . . . . . . . . . 17

(b) Longitudinal residual stresses in the structure middle cross-section obtained by the analytical model. . . . . . 17 
11 Principal stresses in the central transverse section of the plate after forming. . . . . . . . . . . . . . . . . . 17

(a) Numerical principal stresses in the superior surface of the plate, in contact with the stiffener. . . . . . . . . 17

(b) Numerical principal stresses in the inferior surface of the plate. . . . . . . . . . . . . . 17

12 Von Mises stresses in the plate after its forming by springbackforming. . . . . . . . . . . . . . . . 18

(a) Plate's superior surface in contact with the stiffener. . . 18

(b) Plate's inferior surface. . . . . . . . . . . . 18

13 Variation of the plate's curvature radius with respect to the initial tensile stress applied to the stiffener. . . . . . . . . . 19

14 Stiffener's height effect on the plate's curvature radius after forming. . . . . . . . . . . . . . . . . 20

(a) Variation of the curvature radius of the plate's longitudinal center fiber with respect to the stiffener's height. . 20

(b) Variation of the curvature radius's transverse dispersion $\left(\Delta R=R_{\max }-R_{\min }\right)$ in the plate with respect to the stiffener's height. . . . . . . . . . . . 20

15 Plate's width effect on its curvature radius after forming. . . . 21

(a) Variation of the curvature radius of the plate's longitudinal central fiber with respect to the plate's width. . . . 21

(b) Variation of curvature radius's transverse dispersion $(\Delta R=$ $\left.R_{\max }-R_{\min }\right)$ and the radial error in the plate with respect to the plate's width. . . . . . . . . . . . . 21

16 Structure's length effect on the plate's curvature radius after forming. . . . . . . . . . . . . . . . 22

(a) Variation of the curvature radius of the plate's central fiber with respect to the structure's length. . . . . . . . . 22

(b) Variation of the curvature radius's transverse dispersion $\left(\Delta R=R_{\max }-R_{\min }\right)$ in the plate with respect to the structure's length. . . . . . . . . . . . . 22

17 Example of points, measured using the coordinate measuring machine, of a panel formed by springback-forming. (Test 2: plate: $355 \mathrm{~mm} \times 170 \mathrm{~mm} \times 2.4 \mathrm{~mm}$, stiffener: $544 \mathrm{~mm} \times$ $17.6 \mathrm{~mm} \times 2.4 \mathrm{~mm}$, initial applied stress $\left.\sigma^{1}=202.2 \mathrm{MPa}\right) . \quad$. 24 
18 Experimental and numerical transverse variation of longitudinal curvature radius of the three tests' panels, after forming by springback-forming (Test $1: \sigma^{1}=214.2 \mathrm{MPa}$, Test 2: $\sigma^{1}=202.2 \mathrm{MPa}$, Test 3: $\left.\sigma^{1}=155.3 \mathrm{MPa}\right) . \quad \ldots . . . . . .25$

\section{List of Tables}

1 Initial loads and corresponding tensile stresses applied to the stiffener $(4 m \times 17.5 \mathrm{~mm} \times 2.4 \mathrm{~mm})$. . . . . . . . . . . . . . . 19

2 Stiffeners height and the initial tensile stress applied to them in the three experimental tests of springback-forming. . . . . . 23

3 Comparison of curvature radius of the plate's longitudinal center fiber and the transverse dispersion obtained numerically and experimentally from the three tests of springback-forming. 26 